\title{
EXACT EMBEDDING FUNCTORS AND LEFT COHERENT RINGS
}

\author{
KENT R. FULLER AND GEORGE HUTCHINSON
}

(Communicated by Donald Passman)

\begin{abstract}
Let $R$ and $S$ be rings with unit. Suppose $P$ is a free $R$-module on $\beta$ generators, where $\beta$ is an infinite cardinal number not smaller than the cardinality of $R$, and $T$ is the ring of endomorphisms $\operatorname{End}\left({ }_{R} P\right)$.

THEOREM. If $R$ is left coherent and there exists an exact embedding functor $F: R$-Mod $\rightarrow S$-Mod, then ${ }_{S} F(R)_{R}$ is a bimodule such that $F(R)_{R}$ is faithfully flat.

THEOREM. If $F: R$-Mod $\rightarrow S$-Mod is an exact embedding functor, then ${ }_{R} P_{T}$ is a bimodule such that ${ }_{R} P$ is a projective generator (inducing an exact embedding Hom functor from $R$-Mod into $T$-Mod), and ${ }_{S} F(T)_{T}$ is a bimodule such that $F(T)_{T}$ is faithfully flat (inducing an exact embedding tensor product functor ${ }_{S} F(T) \otimes_{T}-$ from $T$-Mod into $S$-Mod).

THEOREM. There exists an exact embedding functor $R$-Mod $\rightarrow S$-Mod iff there exists an $S$-module $N$ and a unit-preserving ring monomorphism $h: \operatorname{End}\left({ }_{R} P\right)$ $\rightarrow \operatorname{End}\left({ }_{s} N\right)$ of their endomorphism rings, such that $h$ preserves and reflects exact pairs of endomorphisms.
\end{abstract}

For $R$ a ring with 1 , let $R$-Mod denote the abelian category of left (unital) $R$-modules and $R$-linear maps, as usual. We are interested in conditions which are equivalent to existence of an exact embedding functor $F: R$-Mod $\rightarrow S$-Mod. In $[5,6]$, several such conditions were given, both for the general case and for the special cases when $R$ is left noetherian or left artinian. In this note, we extend the equivalent condition of existence of a bimodule ${ }_{S} M_{R}$ with $M_{R}$ faithfully flat from the left noetherian case [5, Theorem 2, p. 110] to the left coherent case. We give another equivalent condition for the general case, involving existence of certain homomorphisms of rings of endomorphisms. Also, we show that if there is any exact embedding functor $R$-Mod $\rightarrow S$-Mod, then such a functor can be constructed as the composite of a Hom functor induced by a projective generator and a tensor product functor induced by a faithfully flat module.

All rings will be nontrivial rings with unit, and all ring homomorphisms will preserve units. We will adopt the usual notations $\operatorname{Su}\left({ }_{R} M\right)$ for the complete lattice of $R$-submodules of $M$ in $R$-Mod, $\operatorname{End}\left({ }_{R} M\right)$ for the ring of $R$-linear endomorphisms $\operatorname{Hom}_{R}(M, M)$, and $|X|$ for the cardinality of any set $X$. For $\beta$ a cardinal number, $M^{\beta}$ denotes the Cartesian direct power, and $M^{(\beta)}$ denotes the (possibly infinite)

Received by the editors November 5, 1986 and, in revised form, October 26, 1987. Presented in the earlier version to the American Mathematical Society 93rd Annual Meeting at San Antonio, January 21, 1987 (831-16-268).

1980 Mathematics Subject Classification (1985 Revision). Primary 16A50, 16A65; Secondary $18 \mathrm{E} 20$. 
direct sum. Note that we compose morphisms from left to right $(f: A \rightarrow B$ and $g: B \rightarrow C$ yield $f g: A \rightarrow C)$ in $R$-Mod and $\operatorname{End}\left({ }_{R} M\right)$, and similarly for composition of functors.

1. Definitions AND PROPERTIES. Given $n \geq 1$ and $M$ in $R$-Mod, let $\kappa_{j}: M \rightarrow M^{(n)}$ denote the $j$ th insertion $\kappa_{j}(v)=\left\langle\delta_{j 1} v, \delta_{j 2} v, \ldots, \delta_{j n} v\right\rangle$ (Kronecker delta), and let $\pi_{j}: M^{(n)} \rightarrow M$ denote the $j$ th projection $\pi_{j}\left(v_{1}, v_{2}, \ldots, v_{n}\right)=v_{j}$, $j=1, \ldots, n$. Recall the usual formulas:

1a. $\sum_{j=1}^{n} \pi_{j} \kappa_{j}=I_{n}$ (the identity for $\left.M^{(n)}\right)$. For $j, k \leq n, \kappa_{j} \pi_{k}=0_{M}$ for $j \neq k$, and $\kappa_{j} \pi_{j}=1_{M}$.

2. DEFINITIONS AND PROPERTIES. Recall that a bimodule ${ }_{S} M_{R}$ with $M_{R}$ flat induces an exact functor $H={ }_{S} M_{R} \otimes_{R}-$ from $R$-Mod into $S$-Mod. If $H$ is an exact embedding functor, $M_{R}$ is called faithfully flat.

2a. $F$ is an exact embedding functor $R$-Mod $\rightarrow S$-Mod which preserves infinite direct sums iff $F$ is naturally equivalent to a bimodule tensor product functor ${ }_{S} M_{R} \otimes_{R}$-, where $M_{R}$ is faithfully flat. (This follows from Watts' theorem [8, Theorem 1, p. 5]; see also [5, Theorem 2, p. 110].)

2b. If $F: R$-Mod $\rightarrow S$-Mod is an exact functor, then $F$ is an exact embedding iff $F(R / K) \neq 0$ for all proper (possibly 0) left ideals $K$ of $R$. (See [7, Proposition 7.2, p. 57], and note that there is a nonzero monomorphism $R / K \rightarrow N$ for some $K \neq R$ if $N \neq 0$.)

2c. If $S_{S}$ is a bimodule and $K$ is a left ideal of $R$, then $M \otimes_{R}(R / K) \approx M / M K$ in $S$-Mod (see [1, Exercise 19.1, p. 231]).

It is convenient to introduce some terminology.

3. DEFINITION AND PROPERTIES. An object $A$ of an abelian category $\mathscr{A}$ is called coherent if for each $n \geq 1$ and map $g: A^{(n)} \rightarrow A$, there exists $m \geq 1$ and a map $f: A^{(m)} \rightarrow A^{(n)}$ such that $\langle f, g\rangle$ is exact.

Recall that $R$ is a left coherent ring iff each finitely generated left ideal of $R$ is finitely related (or equivalently, finitely presented); see $[2$, p. 459; 1, p. 229; and 1, Exercise 18.9, p. 214].

3a. $R$ is a left coherent ring iff ${ }_{R} R$ is coherent in $R$-Mod.

In the next result, we show that flat modules can be recovered from coherent objects under certain circumstances. The coherence condition is closely related to the usual characterization of flat modules by linear combinations [1, Lemma 19.19, p. 228]. This connection was observed in [4, Lemma 2.1, p. 534], and the argument below is adapted from [4].

4. Proposition. Suppose $A$ is a coherent object of an abelian category $\mathscr{A}, T$ is the ring of endomorphisms $A \rightarrow A$ in $\mathscr{A}$, and $F: \mathscr{A} \rightarrow S$-Mod is an exact functor for some ring $S$. Then ${ }_{S} F(A)_{T}$ is a bimodule and $F(A)_{T}$ is flat, if $v t=F(t)(v)$ for $v \in F(A)$ and $t \in T$.

ProOF. Assume the hypotheses, and note that ${ }_{S} F(A)_{T}$ is a bimodule for the given scalar product $v t$, using $[1,4.10$, p. 59] and the result that an exact functor preserves endomorphism ring operations and ring units 1 .

To see that $F(A)_{T}$ is flat, suppose that $\sum_{j=1}^{n} v_{j} t_{j}=0$ with $v_{j} \in F(A)$ and $t_{j} \in T, j \leq n$. Then by [1, Lemma 19.19], it suffices to show that there exist $m \geq 1, u_{i} \in F(A)$ for $i \leq m$, and $s_{i j} \in T$ for $i \leq m$ and $j \leq n$, such that 
$\sum_{i=1}^{m} u_{i} s_{i j}=v_{j}$ for $j \leq n$ and $\sum_{j=1}^{n} s_{i j} t_{j}=0$ for $i \leq m$. Let $\kappa_{j}$ and $\pi_{j}$ denote the insertion and projection maps for $A^{(n)}$, and $I_{n}$ the identity map for $A^{(n)}$, as in Definition 1. Let $t: A^{(n)} \rightarrow A$ be the map $\sum_{k=1}^{n} \pi_{k} t_{k}$ of $\mathscr{A}$, so that $\kappa_{j} t=t_{j}$ for $j \leq n$ by 1a. Since $A$ is coherent, there exists $s: A^{(m)} \rightarrow A^{(n)}$ for some $m \geq 1$ such that $\langle s, t\rangle$ is exact, hence $\langle F(s), F(t)\rangle$ is exact. Let $\hat{\kappa}_{i}$ and $\hat{\pi}_{i}$ denote the insertion and projection maps, and $I_{m}$ the identity, for $A^{(m)}, i \leq m$. Define $s_{i j}=\hat{\kappa}_{i} s \pi_{j}$ in $T$ for $i \leq m$ and $j \leq n$. For $i \leq m$, using 1 a and the equation $s t=0$, we have

$$
\sum_{j=1}^{n} s_{i j} t_{j}=\sum_{j=1}^{n} \hat{\kappa}_{i} s \pi_{j} \kappa_{j} t=\hat{\kappa}_{i} s I_{n} t=0 .
$$

Let $v=\sum_{j=1}^{n} F\left(\kappa_{j}\right)\left(v_{j}\right)$ in $F\left(A^{(n)}\right)$. It is convenient to denote function evaluation using reverse order and a binary infix symbol: $x * f$ denotes $f(x)$. Now $v * F\left(\pi_{j}\right)=v_{j}$ for $j \leq n$ by $1 \mathrm{a}$, and

$$
F(t)=F\left(I_{n} t\right)=\sum_{j=1}^{n} F\left(\pi_{j}\right) F\left(\kappa_{j}\right) F(t)=\sum_{j=1}^{n} F\left(\pi_{j}\right) F\left(t_{j}\right),
$$

so that

$$
v * F(t)=\sum_{j=1}^{n}\left(v * F\left(\pi_{j}\right)\right) * F\left(t_{j}\right)=\sum_{j=1}^{n} v_{j} t_{j}=0 .
$$

Since $\operatorname{Im} F(s)=\operatorname{Ker} F(t)$, there exists $u$ in $F\left(A^{(m)}\right)$ such that $u * F(s)=v$, and we define $u_{i}=u * F\left(\hat{\pi}_{i}\right)$ in $F(A)$ for $i \leq m$. Now

$$
\sum_{i=1}^{m} \hat{\pi}_{i} s_{i j}=\sum_{i=1}^{m} \hat{\pi}_{i} \hat{\kappa}_{i} s \pi_{j}=I_{m} s \pi_{j}=s \pi_{j}
$$

for $j \leq n$. Since $(u * F(s)) * F\left(\pi_{j}\right)=v * F\left(\pi_{j}\right)=v_{j}$, we have

$$
\sum_{i=1}^{m} u_{i} s_{i j}=\sum_{i=1}^{m}\left(\left(u * F\left(\hat{\pi}_{i}\right)\right) * F\left(s_{i j}\right)\right)=u * F\left(s \pi_{j}\right)=v_{j}
$$

for $j \leq n$. This proves that $F(A)_{T}$ is flat.

Note that $F(A)_{T}$ in Proposition 4 satisfies a special flatness property: we can define the $s_{i j}$ in the lemma characterizing flat modules depending only on the $t_{j}$, although the $u_{i}$ depend upon both the $t_{j}$ and the $v_{j}$. From this observation, we can obtain a weak converse to Proposition 4. These results are closely related to Chase's theorem [2, Theorem 2.1, p. 460], or see [1, Theorem 19.20, p. 229]: $R$ is left coherent iff $R_{R}^{\beta}$ is flat for all $\beta$ iff every direct product of flat right $R$-modules is flat iff every finitely generated submodule of a free left $R$-module is finitely related.

5. DEFINITION AND PROPERTIES. A module $M_{R}$ is called strongly flat if for each $n \geq 1$ and $t_{j}$ in $R$ for $j \leq n$, there exist $m \geq 1$ and $s_{i j}$ in $R$ for $i \leq m$ and $j \leq n$ such that $\sum_{j=1}^{n} s_{i j} t_{j}=0$ for each $i \leq m$, and if $v_{j} \in M$ for $j \leq n$ such that $\sum_{j=1}^{n} v_{j} t_{j}=0$, then there exist $u_{i}$ in $M$ for $i \leq m$ such that $\sum_{i=1}^{m} u_{i} s_{i j}=v_{j}$ for $j \leq n$.

5a. If $M_{R}$ is strongly flat, then it is flat.

5b. If $\beta$ is any nonzero cardinal number, then $M_{R}$ is strongly flat iff $M_{R}^{(\beta)}$ is strongly flat iff $M_{R}^{\beta}$ is strongly flat. 
5c. If $M_{R}^{\beta}$ is flat for some $\beta \geq \aleph_{0}+|M|$, then $M_{R}$ is strongly flat. (Choose $\tilde{v}_{j}$ in $M^{\beta}$ for $j \leq n$ so that every $n$-tuple $v_{j}$ in $M$ for $j \leq n$ such that $\sum_{j=1}^{n} v_{j} t_{j}=0$ is obtained by projection of $\tilde{v}_{j}$ on some coordinate of $M^{\beta}$. Then $M_{R}$ is strongly flat by $[1,19.19]$ for $\tilde{v}_{j}$.)

5d. Suppose $F: \mathscr{A} \rightarrow S$-Mod is an additive functor of abelian categories, $T=\operatorname{Hom}(A, A)$ for $A$ in $\mathscr{A}$, and $F(A)_{T}$ is given by $v t=F(t)(v)$. If $A$ is coherent in $\mathscr{A}$ and $F$ is an exact functor, then $F(A)_{T}$ is strongly flat (Proposition 4). If $F$ is an additive embedding functor and $F(A)_{T}$ is strongly flat, then $A$ is coherent in $\mathscr{A}$. (The proof, which is like the proof of Proposition 4 , is omitted. Note that $F$ reflects exact sequences by $[3$, Theorem 3.21, p. 66].)

5e. If $R$ is left coherent, then every flat right $R$-module $M_{R}$ is strongly flat. (Apply Chase's theorem and 5c.) If $M_{R}$ is strongly flat and faithfully flat, then $R$ is left coherent. (Use $5 \mathrm{~d}$ and $3 \mathrm{a}$, with $H\left({ }_{R} R\right)_{R} \approx M_{R}$ for $H=M \otimes_{R}$ - from $R$-Mod into Z-Mod, $\mathbf{Z}$ the integers.)

Most of Chase's theorem can be proved by our methods: $R$ is left coherent iff (by 3a) ${ }_{R} R$ is coherent in $R$-Mod iff (by 5 d using the identity functor) $R_{R}$ is strongly flat iff (by $5 \mathrm{~b}, \mathrm{c}$ ) $R_{R}^{\beta}$ is flat for some $\beta \geq \aleph_{0}+|R|$. Also, $R_{R}$ strongly flat implies (by 5b) that $\left(R_{R}^{(\beta)}\right)^{\alpha}$ is flat for all $\alpha$ and $\beta$, which implies (by [1, p. 231]) that every direct product of flat right $R$-modules is flat, which implies that $R_{R}^{\beta}$ is flat for all $\beta$.

We now obtain the first of our equivalence theorems.

6. THEOREM. Suppose $R$ and $S$ are rings with unit, $R$ left coherent, and $F: R$ Mod $\rightarrow S$-Mod is an exact embedding functor. Then ${ }_{S} F(R)_{R}$ is a bimodule such that $F(R)_{R}$ is faithfully flat, so $H={ }_{S} F(R) \otimes_{R}-$ is an exact embedding functor $R$-Mod $\rightarrow S$-Mod which preserves infinite direct sums. Also, $F(C) \approx H(C)$ if $C$ is a finitely related $R$-module.

PROOF. Assuming the hypotheses, ${ }_{S} F(R)_{R}$ is a bimodule and $F(R)_{R}$ is flat, identifying $R$ with $\operatorname{End}\left({ }_{R} R\right)$ and using Propositions 3 a and 4 . So, $H$ is an exact functor which preserves infinite direct sums by $2 \mathrm{a}$.

The remaining parts are obtained essentially by the argument Watts used in [8, Theorem 1]. Suppose $0 \rightarrow A \rightarrow B \rightarrow C \rightarrow 0$ is a short exact sequence in $R$-Mod such that $B \approx R^{(n)}$. Since $F$ and $H$ are right exact, the commutative diagram in $S$-Mod shown below has exact rows:

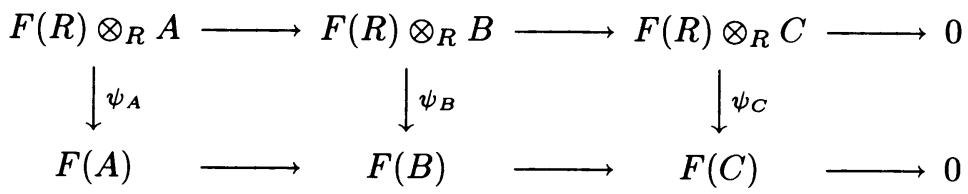

Here, $\psi_{X}: F(R) \otimes_{R} X \rightarrow F(X)$ is given by $\psi_{X}(v \otimes x)=F(r \mapsto r x)(v)$ for $v \in F(R)$, $x \in X$ and $r \in R$, which determines a natural transformation $\psi: H \rightarrow F$. Now $\psi_{B}$ is an isomorphism because $B \approx R^{(n)}$, so $\psi_{C}$ is an epimorphism by the diagram. If $B=R$ and $C=R / A \neq 0$, then $F(C) \neq 0$, hence $H(C) \neq 0$ via $\psi_{C}$. So, $H$ is an exact embedding functor by $2 \mathrm{~b}$. If $C$ is finitely related, so $\psi_{A}$ is an epimorphism like $\psi_{C}$ because $A$ is finitely generated also, then $\psi_{C}$ is an isomorphism by the Five Lemma $[1,3.15(1)$, p. 50], and $F(C) \approx H(C)$. 
The remaining results depend upon recalling the special properties of the endomorphism rings $\operatorname{End}\left({ }_{R} R^{(\beta)}\right)$ for free $R$-modules with $\beta$ generators, where $\beta$ is a sufficiently large infinite cardinal number.

7. DEFINITIONS AND PROPERTIES. Let $R$ be a ring, let $\beta$ be an infinite cardinal number with $\beta \geq|R|$, and let $T$ denote $\operatorname{End}\left({ }_{R} R^{(\beta)}\right)$. Let $B=\left\{e_{\nu}\right\}_{\nu<\beta}$ be a set of free generators for ${ }_{R} R^{(\beta)}$, indexed by the ordinal numbers $\nu<\beta$.

7a. If $M$ is a submodule of ${ }_{R} R^{(\beta)}$, there is an endomorphism $t$ in $T$ such that $\operatorname{Im} t=M$ (since $\left|{ }_{R} R^{(\beta)}\right|=\beta$ because $\beta \geq \aleph_{0}+|R|$, and ${ }_{R} R^{(\beta)}$ is free on $\beta$ generators).

7b. The principal left ideals $\{T t: t \in T\}$ of $T$ form a sublattice of the lattice $\mathrm{Su}\left({ }_{T} T\right)$ of left ideals of $T$, and this sublattice is isomorphic to $\operatorname{Su}\left({ }_{R} R^{(\beta)}\right)$ via the map $T t \mapsto \operatorname{Im} t$. (Use 7a, noting that $T t=\{u \in T: \operatorname{Im} u \leq \operatorname{Im} t\}$.) Similarly, $\mathrm{Su}\left({ }_{T} T\right)$ is isomorphic to the lattice of lattice ideals of $\operatorname{Su}\left({ }_{R} R^{(\bar{\beta})}\right)$.

7c. $T$ is left coherent. (By $7 \mathrm{~b}$, any finitely generated left ideal of $T$ equals $T w$ for some $w$ in $T$, hence there is an epimorphism $T \rightarrow T w$ given by $t \mapsto t w$, which has a finitely generated kernel $T u \rightarrow T$ where $u$ with $\operatorname{Im} u=\operatorname{Ker} w$ exists by 7a.)

7d. ${ }_{R} R_{T}^{(\beta)}$ is a bimodule [1, Proposition 4.10 , p. 59] such that ${ }_{R} R^{(\beta)}$ is a projective generator. Therefore, $G=\operatorname{Hom}_{R}\left({ }_{R} R_{T}^{(\beta)},-\right)$ is an exact embedding functor from $R$-Mod into $T$-Mod. Note that $G$ does not preserve infinite direct sums in general.

7e. $\phi: R \rightarrow T$ given by $\phi(r)\left(e_{\nu}\right)=r e_{\nu}$ for $r \in R$ and $\nu<\beta$ is a ring homomorphism preserving 1 . So, $\phi$ induces an exact embedding functor $H_{\phi}: T$-Mod $\rightarrow R$ Mod by change of rings $\left(r v=\phi(r) v\right.$ for $v \in{ }_{T} M$ and $\left.r \in R\right)$.

7f. If $P={ }_{R} R^{(\beta)}$, then for each $n \geq 1, P \approx P^{(n)}$ in $R$-Mod. (Partition the free generating set $B$ for $R^{(\beta)}$ into subsets $B_{1}, B_{2}, \ldots, B_{n}$, each of cardinality $\beta$, and let $P_{i}$ denote the submodule of $P$ generated by $B_{i}$. Show that $P_{1} \oplus P_{2} \oplus \cdots \oplus P_{n}$ is an internal direct sum for $P$ such that $P \approx P_{i}$ for each $i \leq n$.)

7g. ${ }_{R} R^{(\beta)}$ is coherent in $R$-Mod. (Use 7a and 7f.)

The second equivalence result is now obtained.

8. THEOREM. Suppose $R$ and $S$ are rings with unit such that there exists an exact embedding functor $F: R$-Mod $\rightarrow S$-Mod. If $\beta \geq \aleph_{0}+|R|$ and $T=$ $\operatorname{End}\left({ }_{R} R^{(\beta)}\right)$, then there exist exact embedding functors

$$
G: R \text {-Mod } \rightarrow T \text {-Mod with } G=\operatorname{Hom}_{R}\left({ }_{R} R_{T}^{(\beta)},-\right),
$$

and

$$
H: T \text {-Mod } \rightarrow S \text {-Mod with } H={ }_{S} F\left({ }_{R} T_{T}\right) \otimes_{T}-.
$$

If $|M| \leq \beta$ for $M$ in $R$-Mod, then $H(G(M)) \approx F\left(M^{\beta}\right)$.

PROOF. For $G$, use $7 \mathrm{~d}$. By $7 \mathrm{e}$, we have an exact embedding functor $H_{\phi}: T$ Mod $\rightarrow R$-Mod. So, we obtain $H$ by applying $7 \mathrm{c}$ and Theorem 6 to $H_{\phi} F$, observing that $H_{\phi}\left({ }_{T} T_{T}\right)={ }_{R} T_{T}$. Suppose $|M| \leq \beta$ for $M$ in $R$-Mod, so there exists $0 \rightarrow K \rightarrow R^{(\beta)} \rightarrow M \rightarrow 0$ exact in $R$-Mod. Now $G\left(R^{(\beta)}\right)=T T$, so $G(M)$ is finitely generated. But $G(K)$ is finitely generated similarly, so $G(M)$ is finitely related in $T$-Mod. By Theorem 6 then,

$$
H(G(M))={ }_{S} F\left({ }_{R} T_{T}\right) \otimes_{T} G(M) \approx F\left(H_{\phi}(G(M))\right) \approx F\left(M^{\beta}\right),
$$

since $H_{\phi}(G(M)) \approx{ }_{R} M^{\beta}$ using $[1,16.4$, p. 181]. 
Let $P={ }_{R} R^{(\beta)}$ for $\beta \geq \aleph_{0}+|R|$. Then $P \approx P^{(n)}$ for each $n \geq 1$ by 7 f, and $P$ is coherent in $R$-Mod by $7 \mathrm{~g}$. Observe that $f$ and $g$ in Definition 3 for $P$ can be regarded as maps $P \rightarrow P$. Therefore, we only need the ring of endomorphisms of $P$ to carry through the argument proving flatness in Proposition 4. After some preparation, we show that this leads to our final equivalence theorem.

9. DEFINITIONS AND PROPERTIES. For $M$ in $R$-Mod and $N$ in $S$-Mod, a ring homomorphism $h: \operatorname{End}\left({ }_{R} M\right) \rightarrow \operatorname{End}\left({ }_{S} N\right)$ is said to preserve exactness if for $c, d$ in $\operatorname{End}\left({ }_{R} M\right)$ such that $\langle c, d\rangle$ is exact in $R$-Mod, $\langle h(c), h(d)\rangle$ is exact in $S$-Mod. We say that $h$ reflects exactness if $\langle h(c), h(d)\rangle$ exact in $S$-Mod implies $\langle c, d\rangle$ is exact in $R$-Mod.

9a. If $R$ and $S$ are rings with unit and $F: R$-Mod $\rightarrow S$-Mod is an exact functor, then for each $M$ in $R$-Mod, $F$ induces a ring homomorphism $F_{M}: \operatorname{End}\left({ }_{R} M\right) \rightarrow$ End $\left({ }_{S} F(M)\right)$ which preserves exactness. If $F$ is an exact embedding functor, then each $F_{M}$ is a ring monomorphism which preserves and reflects exactness (use [3, Theorem 3.21, p. 66]).

10. THEOREM. For rings $R$ and $S$ with 1 , the following are equivalent:

10a. There exists an exact embedding functor $F: R$-Mod $\rightarrow S$-Mod.

$10 \mathrm{~b}$. For some infinite cardinal number $\beta \geq|R|$ and $N$ in $S$-Mod, there exists a (unit-preserving) ring monomorphism $h: \operatorname{End}\left({ }_{R} R^{(\beta)}\right) \rightarrow \operatorname{End}\left({ }_{S} N\right)$ which preserves and reflects exactness.

PrOOF. Clearly $10 \mathrm{a} \Rightarrow 10 \mathrm{~b}$ by $9 \mathrm{a}$. So, we assume $10 \mathrm{~b}$ and prove $10 \mathrm{a}$. Define $F: R$-Mod $\rightarrow S$-Mod to be the composite functor

$$
R \text {-Mod } \stackrel{G}{\longrightarrow} T \text {-Mod } \stackrel{H}{\longrightarrow} S \text {-Mod }
$$

where $G$ denotes $\operatorname{Hom}_{R}\left({ }_{R} R_{T}^{(\beta)},-\right)$ as in $7 \mathrm{~d}$, and $H$ denotes ${ }_{S} N_{T} \otimes_{T}$-, with $N_{T}$ given by $v t=h(t)(v)$ for $t \in T$ and $v \in N$. The argument that ${ }_{S} N_{T}$ is a bimodule with $N_{T}$ flat is similar to the proof of Proposition 4, using an adaptation employing $7 \mathrm{f}$ and $7 \mathrm{~g}$. Therefore, $G$ and $H$ are exact functors, and hence so is $F$.

Let $K$ be a proper left ideal of $R$, and let $K_{0}=K e_{0} \vee P_{0}$ in $\operatorname{Su}\left({ }_{R} R^{(\beta)}\right)$, where $P_{0}$ is generated by $\left\{e_{\nu}: 0<\nu<\beta\right\}$. By 7 a, there exists $w$ in $T$ such that $\operatorname{Im} w=K_{0}$. Since $t \in T w$ iff $\operatorname{Im} t \leq \operatorname{Im} w$ by $7 \mathrm{~b}$, there is a $T$-linear monomorphism $\lambda: T / T w \rightarrow$ $G(R) / G(K)$ such that

$$
\lambda(1+T w)=\pi_{0}+G(K),
$$

where $\pi_{0}$ in $G(R)=\operatorname{Hom}_{R}\left({ }_{R} R_{T}^{(\beta)},{ }_{R} R\right)$ is given by $\pi_{0}\left(e_{0}\right)=1$ and $\pi_{0}\left(e_{\nu}\right)=0$ for $0<\nu<\beta$. Now $\operatorname{Im} w=K_{0} \neq P$, so $\left\langle w, 0_{P}\right\rangle$ is not exact in $R$-Mod, and so $\left\langle h(w), 0_{N}\right\rangle$ is not exact in $S$-Mod because $h$ reflects exactness. Then $N T w=$ $N w=\operatorname{Im} h(w) \neq \operatorname{Ker} 0_{N}=N$. So ${ }_{S} N \otimes_{T}(T / T w) \approx N / N T w \neq 0$ by $2 \mathrm{c}$, and

$$
F(R / K)=H(G(R / K)) \approx{ }_{S} N \otimes_{T}(G(R) / G(K)) \neq 0
$$

via the monomorphism $N \otimes_{T} \lambda$. By $2 \mathrm{~b}$ then, $F$ is an exact embedding functor.

Now $N_{T}$ may not be faithfully flat for ${ }_{S} N_{T}$ induced by $h$ above, but we can find ${ }_{S} M_{T}$ with $M_{T}$ faithfully flat by Theorem 8 .

Does existence of an exact embedding functor $R$-Mod $\rightarrow S$-Mod always lead to existence of a bimodule ${ }_{S} M_{R}$ such that $M_{R}$ is faithfully flat? By $2 \mathrm{a}, 7 \mathrm{~d}$ and 
Theorem 8, this reduces to the following:

Open problem. Suppose $\mathrm{S} R$ is a ring and $T=\operatorname{End}\left({ }_{R} R^{(\beta)}\right)$ for $\beta$ an infinite cardinal number. Does there always exist a bimodule ${ }_{T} M_{R}$ such that $M_{R}$ is faithfully flat?

For $R$ left coherent, ${ }_{T} G(R)_{R}$ is such a bimodule by $7 \mathrm{~d}$ and Theorem 6 . Note that $G(R)_{R}=\operatorname{Hom}_{R}\left({ }_{R} R^{(\beta)},{ }_{R} R_{R}\right) \approx R_{R}^{\beta}$ by $[1,16.4$, p. 181$]$.

\section{REFERENCES}

1. F. Anderson and K. Fuller, Rings and categories of modules, Springer-Verlag, Berlin, Heidelberg and New York, 1974.

2. S. Chase, Direct products of modules, Trans. Amer. Math. Soc. 97 (1960), 457-473.

3. P. Freyd, Abelian categories: An introduction to the theory of functors, Harper and Row, New York, 1964.

4. K. Fuller, Density and equivalence, J. Algebra 29 (1974), 528-550.

5. G. Hutchinson, Exact embedding functors between categories of modules, J. Pure Appl. Algebra 25 (1982), 107-111.

6. __ Addendum to "Exact embedding functors between categories of modules", J. Pure Appl. Algebra 45 (1987), 99-100.

7. B. Mitchell, Theory of categories, Academic Press, New York, 1965.

8. C. Watts, Intrinsic characterizations of some additive functors, Proc. Amer. Math. Soc. 11 (1960), 5-8.

DePARTMENT OF MAThematics, UNiversity of IOWA, IOWA City, IOWA 52242

Division OF COMPUTER RESEARCH AND TEChNOLOgY, NATIONAL INSTITUTES OF HEALTH, BETHESDA, MARYLAND 20892 